Article

\title{
Interval Grey Prediction Models with Forecast Combination for Energy Demand Forecasting
}

\author{
Peng Jiang ${ }^{1}\left(\mathbb{D}\right.$, Yi-Chung $\mathrm{Hu}^{2, *} \mathbb{D}$, Wenbao Wang ${ }^{3, *}$, Hang Jiang ${ }^{4}\left(\mathbb{D}\right.$ and Geng $\mathrm{Wu}^{2}$ \\ 1 School of Business, Shandong University, Weihai 264209, China; jiangpeng1006@sdu.edu.cn \\ 2 Department of Business Administration, Chung Yuan Christian University, Taoyuan City 32023, Taiwan; \\ g10804108@cycu.edu.tw \\ 3 College of Civil Engineering, Yango University, Fuzhou 350015, China \\ 4 School of Business Administration, Jimei University, Xiamen 361021, China; hangjiang@jmu.edu.cn \\ * Correspondence: ychu@cycu.edu.tw (Y.-C.H.); wangwb@ygu.edu.cn (W.W.)
}

Received: 22 May 2020; Accepted: 10 June 2020; Published: 11 June 2020

check for updates

\begin{abstract}
Time series data for decision problems such as energy demand forecasting are often derived from uncertain assessments, and do not meet any statistical assumptions. The interval grey number becomes an appropriate representation for an uncertain and imprecise observation. In order to obtain nonlinear interval grey numbers with better forecasting accuracy, this study proposes a combined model by fusing interval grey numbers estimated by neural networks (NNs) and the grey prediction models. The proposed model first uses interval regression analysis using NNs to estimate interval grey numbers for a real valued sequence; and then a grey residual modification model is constructed using the upper and lower wrapping sequences obtained by NNs. It turns out that two different kinds of interval grey numbers can be estimated by nonlinear interval regression analysis. Forecasting accuracy on real data sequences was then examined by the best non-fuzzy performance values of the combined model. The proposed combined model performed well compared with the other interval grey prediction models considered.
\end{abstract}

Keywords: forecast combination; neural network; interval regression; grey number; energy demand

\section{Introduction}

A grey number refers to an inexact number, which becomes an interval grey number if its interval, including upper and lower limits, can be found [1]. Available information on many time series problems, such as energy demand forecasting, is often derived from uncertain assessments, and do not meet any statistical assumptions [2-4]. To deal with uncertainty, interval grey numbers can be a proper form of representing those uncertain and imprecise observations [1,5-7]. To estimate interval grey numbers, interval regression analysis is a reasonable technique, as it has a high capability of processing uncertain data [8,9]. In particular, because neural networks (NNs) have a high capability to represent nonlinear mappings, the wrapping sequences comprising upper and lower limits can be effectively determined using two NNs [8,10-13]. It is noted that the best non-fuzzy performance value (BNP)—an average of the upper and lower limits for each sample — can be used to verify the forecasting accuracy of an interval model $[6,14]$.

Previously, since the grey prediction per se has the advantage of using limited samples for model fitting without meeting any statistical assumptions [4,15-18], a few studies on the basis of the frequently-used GM(1,1) were proposed to predict interval grey numbers, including the interval grey number prediction model (IGNPM) [19], the IGNPM based on DGM(1,1) [20], the grey number grey modification model $(\operatorname{GGMM}(1,1))[1]$ and a single variable discrete grey forecasting model (IN-DGM) [6]. Furthermore, it has been shown that the forecasting accuracy of the GM(1,1) can be 
improved by residual modification [21-23], which employs predicted residuals to revise predicted values obtained from the $\mathrm{GM}(1,1)$. Nevertheless, most previous residual modification models have focused on residual sign estimation [3,24-26] (Hsu, 2003; Hsu and Chen, 2003; and Hsu and Wen, 1998). To solve this problem, the FLNGM(1,1), using functional link nets (FLNs), was presented by $\mathrm{Hu}$ [27] to improve the forecasting accuracy for energy demand forecasting by estimating the modification range of each predicted residual.

Undoubtedly, because of the increasing importance of global energy consumption, the prediction of energy demand is a significant issue for energy management and environmental protection [4]. In light of the distinctive features of nonlinear interval regression analysis and the $\operatorname{FLNGM}(1,1)$, this study addresses the development of a nonlinear interval model by combining these two prediction models to effectively forecast energy demand. Furthermore, the fusion of forecasts from different prediction models can be helpful in improving prediction accuracy [28-30]. In this realization, based on the upper and lower wrapping sequences determined by NNs, it is interesting to further apply the FLNGM $(1,1)$ to predict the developing trends of upper and lower limits. This ends up with a nonlinear interval model consisting of two different kinds of estimated interval grey numbers: one is based on NNs, and the other is based on the $\operatorname{FLNGM}(1,1)$. To verify the forecasting accuracy, the proposed combined model, NN-FLNGM $(1,1)$, fuses the BNP values obtained from NNs with FLNGM $(1,1)$ for nonlinear interval regression analysis.

The rest of the paper is organized as follows. Section 2 briefly introduces interval grey numbers determined by neural networks. Section 3 demonstrates the current GM(1,1) and FLNGM $(1,1)$ models, while Section 4 details the proposed NN-FLNGM $(1,1)$ model. Section 5 examines prediction accuracy for the proposed model using real data of energy demand, and Section 6 summarizes the outcomes and concludes the paper.

\section{Interval Grey Number Forecasting Using Neural Networks}

Ishibuchi and Tanaka [13] applied two $\mathrm{NNs}, \mathrm{NN}_{u}$ and $\mathrm{NN}_{l}$, to perform interval regression analysis, where $\mathrm{NN}_{u}$ determined the upper limits and $\mathrm{NN}_{l}$ determined the lower limits of a nonlinear interval model. The principle of their approach was that interval grey numbers can be derived from two NNs. For simplicity, we denote such an interval grey number forecasting model using two NNs as IGF-NNs.

\subsection{Nonlinear Interval Regression Analysis}

For a sequence $\mathbf{x}^{(0)}=\left(x_{1}^{(0)}, x_{2}^{(0)}, \ldots, x_{n}^{(0)}\right)=\left(y_{1}, y_{2}, \ldots, y_{n}\right)$, let $\left(t_{1}, y_{1}\right),\left(t_{2}, y_{2}\right), \ldots,\left(t_{n}, y_{n}\right)$ comprise a model-fitting data set for $\mathrm{NN}_{u}$ and $\mathrm{NN}_{l}$, where $\left(t_{p}, y_{p}\right)(p=1,2, \ldots, n)$ is the input-output pattern at the $p$-th time point, $t_{p}$. Let $g_{u}\left(t_{p}\right)$ and $g_{l}\left(t_{p}\right)$ be the output functions from $\mathrm{NN}_{u}$ and $\mathrm{NN}_{l}$, respectively. A nonlinear optimization problem can be formulated to determine a nonlinear interval model as follows:

$$
\begin{gathered}
\text { Minimize }\left(g_{u}\left(t_{1}\right)-g_{l}\left(t_{1}\right)\right)+\left(g_{u}\left(t_{2}\right)-g_{l}\left(t_{2}\right)\right)+\ldots+\left(g_{u}\left(t_{m}\right)-g_{l}\left(t_{m}\right)\right) \\
\text { subject to } g_{l}\left(t_{p}\right) \leq y_{p} \leq g_{u}\left(t_{p}\right), p=1,2, \ldots, n
\end{gathered}
$$

where $\left(g_{u}\left(t_{p}\right)-g_{l}\left(t_{p}\right)\right)$ denotes the estimated interval width at $t_{p}$.

Therefore, the above problem addresses the determination of the nonlinear interval model with the minimum sum of the predicted interval widths, subject to the condition that the estimated data interval includes all the given patterns. In particular, Ishibuchi and Tanaka [13] derived two simple algorithms to determine $g_{u}(t)$ and $g_{l}(t)$, which approximately satisfied the constraint condition. That is, interval grey numbers can be estimated by a nonlinear interval model established by two NNs. 


\subsection{Determining Upper and Lower Limits}

The cost function, $E_{u}$, with weighting scheme, $\omega_{p}$, was used to determine the upper limit $g_{u}(t)$,

$$
E_{u}=\sum_{p=1}^{m} \frac{1}{2} \omega_{p}\left(y_{p}-g_{u}\left(t_{p}\right)\right)^{2}
$$

where:

$$
\omega_{p}=\left\{\begin{array}{l}
1, \text { if } y_{p}>g_{u}\left(t_{p}\right) \\
\omega, \text { if } y_{p} \leq g_{u}\left(t_{p}\right)
\end{array}\right.
$$

To determine $g_{l}(t)$, we used the cost function $E_{l}$ as

$$
E_{l}=\sum_{p=1}^{m} \frac{1}{2} \omega_{p}\left(y_{p}-g_{l}\left(t_{p}\right)\right)^{2}
$$

where

$$
\omega_{p}=\left\{\begin{array}{l}
1, \text { if } y_{p}<g_{l}\left(t_{p}\right) \\
\omega, \text { if } y_{p} \geq g_{l}\left(t_{p}\right)
\end{array}\right.
$$

and $\omega$ is a small positive value in $(0,1)$. The learning rule for each connection weight can be derived by the gradient descent. The two algorithms regarding $g_{u}(t)$ and $g_{l}(t)$ are the same aside from the weighting schemes. For simplicity, we omit the learning rules here.

Defuzzification, which locates the BNP, provides decision makers with concrete estimations [31] rather than intervals. Since $g_{u}$ and $g_{l}$ constitute the IGF-NNs, its BNP denoted $x_{k \mathrm{NN}}^{(0)}$ is viewed as a predicted value of $x_{k}^{(0)}$, and can be the center of both limits (Sun et al., 2016; Xie et al., 2014),

$$
x_{k^{\mathrm{NN}}}^{(0)}=1 / 2\left(g_{u}(k)+g_{l}(k)\right), k=1,2, \ldots, n
$$

\section{3. $\operatorname{GM}(1,1)$ and FLNGM(1,1)}

As mentioned above, the residual modification mechanism can be employed to improve the forecasting accuracy of the original $\operatorname{GM}(1,1)$. The $\operatorname{FLNGM}(1,1)$ is a kind of residual modification model, and was successfully applied to forecast energy demand. To build the $\operatorname{FLNGM}(1,1)$, two grey prediction models can be set up consecutively; the original $\mathrm{GM}(1,1)$ is set up first, followed by its residual one.

\section{1. $G M(1,1)$}

Regarding a sequence $\mathbf{x}^{(0)}=\left(x_{1}^{(0)}, x_{2}^{(0)}, \ldots, x_{n}^{(0)}\right), \mathbf{x}^{(1)}=\left(x_{1}^{(1)}, x_{2}^{(1)}, \ldots, x_{n}^{(1)}\right)$ is newly generated by the accumulated generating operation (AGO)

$$
x_{k}^{(1)}=\sum_{j=1}^{k} x_{k}^{(0)}, k=1,2, \ldots, n
$$

and a first-order differential equation that can be used to approximate $x_{1}^{(1)}, x_{2}^{(1)}, \ldots, x_{n}^{(1)}$ is:

$$
\frac{d x^{(1)}}{d t}+a x^{(1)}=b
$$

The solution of the above differential equation is

$$
\hat{x}_{k}^{(1)}=\left(x_{1}^{(0)}-\frac{b}{a}\right) e^{-a(k-1)}+\frac{b}{a}
$$


where $a$ is the developing coefficient and $b$ is the control variable. Then, $a$ and $b$ are further estimated using the grey difference equations

$$
x_{k}^{(0)}+a z_{k}^{(1)}=b, k=2,3, \ldots, n
$$

where $z_{k}^{(1)}$ is formulated as

$$
z_{k}^{(1)}=\alpha x_{k}^{(1)}+(1-\alpha) x_{k-1}^{(1)}
$$

where usually $\alpha=0.5$. The ordinary least-squares method (OLS) are involved in parameter estimation,

$$
\left[\begin{array}{l}
a \\
b
\end{array}\right]=\left(\mathbf{B}^{T} \mathbf{B}\right)^{-1} \mathbf{B}^{T} \mathbf{y}
$$

where

$$
\mathbf{B}=\left[\begin{array}{cc}
-z_{2}^{(1)} & 1 \\
-z_{3}^{(1)} & 1 \\
\vdots & \vdots \\
-z_{n}^{(1)} & 1
\end{array}\right]
$$

and:

$$
\mathbf{y}=\left[x_{2}^{(0)}, x_{3}^{(0)}, \ldots, x_{n}^{(0)}\right]^{T}
$$

Finally, the predicted value is derived by the inverse AGO (IAGO):

$$
\hat{x}_{k}^{(0)}=\hat{x}_{k}^{(1)}-\hat{x}_{k-1}^{(1)}, k=2,3, \ldots, n
$$

Therefore,

$$
\hat{x}_{k}^{(0)}=\left(1-e^{a}\right)\left(x_{1}^{(0)}-\frac{b}{a}\right) e^{(k-1)}, k=2,3, \ldots, n
$$

and $\hat{x}_{1}^{(1)}=\hat{x}_{1}^{(0)}$ holds.

\section{2. $\operatorname{FLNGM}(1,1)$}

Let $\varepsilon^{(0)}=\left(\varepsilon_{2}^{(0)}, \varepsilon_{3}^{(0)}, \ldots, \varepsilon_{n}^{(0)}\right)$, where

$$
\varepsilon_{k}^{(0)}=\left|x_{k}^{(0)}-\hat{x}_{k}^{(0)}\right|, k=2,3, \ldots, n
$$

a residual $\mathrm{GM}(1,1)$ can be established by $\varepsilon^{(0)}$, and the predicted value is:

$$
\hat{\varepsilon}_{k}^{(0)}=\left(1-e^{a_{\varepsilon}}\right)\left(\varepsilon_{2}^{(0)}-\frac{b_{\varepsilon}}{a_{\varepsilon}}\right) e^{-a_{\varepsilon}(k-1)}, k=3,4, \ldots, n
$$

Let $w_{j}(j=1,2, \ldots, 5)$ be the connection weights, and $\theta$ be a bias. By presenting $\left(t_{k}, \sin \left(\pi t_{k}\right)\right.$, $\left.\cos \left(\pi t_{k}\right), \sin \left(2 \pi t_{k}\right), \cos \left(2 \pi t_{k}\right)\right)$ to an FLN, its actual output is computed as:

$$
y_{k}=\tanh \left(w_{1} t_{k}+w_{2} \sin \left(\pi t_{k}\right)+w_{3} \cos \left(\pi t_{k}\right)+w_{4} \sin \left(2 \pi t_{k}\right)+w_{5} \cos \left(2 \pi t_{k}\right)+\theta\right)
$$

where $-1 \leq y_{k} \leq 1$, and tanh is an activation function formulated as:

$$
\tanh (z)=\frac{e^{z}-e^{-z}}{e^{z}+e^{-z}}
$$


A new predicted value is computed as

$$
\hat{x}_{k^{\mathrm{FN}}}^{(0)}=\hat{x}_{k}^{(0)}+3 y_{k} \hat{\varepsilon}_{k}^{(0)}, k=2,3, \ldots, n
$$

This signifies that the maximum amount of adjusting $\hat{x}_{k}^{(0)}$ is $3 \hat{\varepsilon}_{k}^{(0)}$, while $y_{k}$ with a positive or negative sign can moderate the impact of the amount on $\hat{x}_{k}^{(0)}$. A genetic algorithm (GA) can be applied to determine optimal values of $w_{j}$ and $\theta$ to build an $\operatorname{FLNGM}(1,1)$ with high accuracy. For simplicity, the details can be found in $\mathrm{Hu}$ [27].

\section{The Proposed Combined Prediction Model}

To build the proposed NN-FLNGM $(1,1)$, the first step is to find interval grey numbers using two neural networks, followed by the setting up of $\operatorname{FLNGM}(1,1)$, and then determining the BNP for the combined model. Section 4.1 describes the construction of the $\operatorname{FLNGM}(1,1)$ for interval grey number forecasting (IGF-FLNGM(1,1)), and Section 4.2 describes the evaluation of prediction accuracy.

\subsection{Constructing the FLNGM(1,1) for Interval Grey Number Forecasting}

After training $\mathrm{NN}^{*}$ and $\mathrm{NN}^{*}$, two new data sequences can be created: the upper wrapping sequence $\mathbf{x}_{u}^{(0)}$ by $\mathrm{NN}^{*}$ and the lower wrapping sequence $\mathbf{x}_{l}^{(0)}$ by $\mathrm{NN}_{*}$, where $\boldsymbol{x}_{u}^{(0)}=\left(g_{u}\left(t_{1}\right), g_{u}\left(t_{2}\right), \ldots\right.$, $\left.g_{u}\left(t_{n}\right)\right)=\left(x_{u, 1}^{(0)}, x_{u, 2}^{(0)}, \ldots, x_{u, n}^{(0)}\right)$, and $\mathbf{x}_{l}^{(0)}=\left(g_{l}\left(t_{1}\right), g_{l}\left(t_{2}\right), \ldots, g_{l}\left(t_{n}\right)\right)=\left(x_{l, 1}^{(0)}, x_{l, 2}^{(0)}, \ldots, x_{l, n}^{(0)}\right)$. Consequently, estimation of each available sample can be automatically extended from a single point to an interval. Then two $\operatorname{FLNGM}(1,1)$ can be set up for the IGF-FLNGM $(1,1)$ : one being the upper $\operatorname{FLNGM}(1,1)$, and the other the lower $\operatorname{FLNGM}(1,1)$. Using $\mathbf{x}_{u}^{(0)}$, the predicted value, $\hat{x}_{u, k^{\mathrm{FLN}}}^{(0)}$ produced by the upper $\operatorname{FLNGM}(1,1)$ is

$$
\hat{x}_{u, k^{\mathrm{FLN}}}^{(0)}=\hat{x}_{u, k}^{(0)}+3 y_{u, k} \hat{\varepsilon}_{u, k}^{(0)}, k=2,3, \ldots, n
$$

where $-1 \leq y_{u, k} \leq 1$, and $\hat{\varepsilon}_{u, k}^{(0)}$ is:

$$
\hat{\varepsilon}_{u, k}^{(0)}=\left(1-e^{a_{u, \varepsilon}}\right)\left(\varepsilon_{u, 2}^{(0)}-\frac{b_{u, \varepsilon}}{a_{u, \varepsilon}}\right) e^{-a_{u, \varepsilon}(k-1)}, k=3,4, \ldots, n
$$

Using $\mathbf{x}_{l}^{(0)}$, the predicted value $\hat{x}_{l, k^{\mathrm{FLN}}}^{(0)}$ produced by the $\operatorname{IGF-FLNGM}(1,1)$ for the lower limits

$$
\hat{x}_{l, k^{\mathrm{FLN}}}^{(0)}=\hat{x}_{l, k}^{(0)}+3 y_{l, k} \hat{\varepsilon}_{l, k}^{(0)}, k=2,3, \ldots, n
$$

where $-1 \leq y_{l, k} \leq 1$, and $\hat{\varepsilon}_{l, k}^{(0)}$ is:

$$
\hat{\varepsilon}_{l, k}^{(0)}=\left(1-e^{a_{l, \varepsilon}}\right)\left(\varepsilon_{l, 2}^{(0)}-\frac{b_{l, \varepsilon}}{a_{l, \varepsilon}}\right) e^{-a_{l, \varepsilon}(k-1)}, k=3,4, \ldots, n
$$

The BNP value for $x_{k}^{(0)}$ can be formulated as:

$$
\widetilde{x}_{k^{F L N}}^{(0)}=\frac{1}{2}\left(\hat{x}_{u, k^{\mathrm{FLN}}}^{(0)}+\hat{x}_{l, k^{\mathrm{FLN}}}^{(0)}\right), k=1,2, \ldots, n
$$




\subsection{Prediction Performance Evaluation}

Let the BNP for the combined model for $x_{k}^{(0)}$ be denoted by $\widetilde{x}_{k^{c o m}}^{(0)}$. Although both $x_{k^{\mathrm{NN}}}^{(0)}$ and $\widetilde{x}_{k^{F L N}}^{(0)}$ can be used to compute $\widetilde{x}_{k^{c o m}}^{(0)}$, the extent to which $x_{k^{N N}}^{(0)}$ and $\widetilde{x}_{k^{F L N}}^{(0)}$ contribute can differ. Based on the forecasting accuracy of IGF-NNs and IGF-FLNGM $(1,1), \widetilde{x}_{k^{c o m}}^{(0)}$ is defined as

$$
\widetilde{x}_{k^{\mathrm{com}}}^{(0)}=w_{1} x_{k^{\mathrm{NN}}}^{(0)}+w_{2} \widetilde{x}_{k^{F L N}}^{(0)}
$$

where $w_{1}$ and $w_{2}$ are the relative weights of $x_{k^{\mathrm{NN}}}^{(0)}$ and $\widetilde{x}_{k^{F L N}}^{(0)}$, respectively, and

$$
w_{1}=\frac{\left(\frac{1}{\text { MAPE }_{\mathrm{NN}}}\right)^{\alpha}}{\left(\frac{1}{\mathrm{MAPE}_{\mathrm{NN}}}\right)^{\alpha}+\left(\frac{1}{\mathrm{MAPE}_{\mathrm{FLNGM}(1,1)}}\right)^{\beta}}
$$

and

$$
w_{2}=\frac{\left(\frac{1}{\operatorname{MAPE}_{\mathrm{FLNGM}(1,1)}}\right)^{\beta}}{\left(\frac{1}{\mathrm{MAPE}_{\mathrm{NN}}}\right)^{\alpha}+\left(\frac{1}{\operatorname{MAPE}_{\mathrm{FLNGM}(1,1)}}\right)^{\beta}}
$$

in which $\alpha$ and $\beta$ are positive real numbers, and MAPE denotes the mean absolute percentage error (MAPE).

For the IGF-NNs, its forecasting accuracy measured by the MAPE is defined as:

$$
\mathrm{MAPE}_{\mathrm{NN}}=\frac{1}{n} \sum_{k=1 \ldots n} \frac{\left|x_{k}^{(0)}-\widetilde{x}_{k^{N N}}^{(0)}\right|}{x_{k^{\mathrm{NN}}}^{(0)}} \times 100 \%
$$

For the IGF-FLNGM $(1,1)$, its MAPE is:

$$
\operatorname{MAPE}_{\mathrm{FLNGM}(1,1)}=\frac{1}{n} \sum_{k=1 \ldots n} \frac{\left|x_{k}^{(0)}-\widetilde{x}_{k^{F L N}}^{(0)}\right|}{x_{k}^{(0)}} \times 100 \%
$$

As a result, the MAPE of the NNs-FLNGM(1,1) is formulated as:

$$
\mathrm{MAPE}_{\mathrm{com}}=\frac{1}{n} \sum_{k=1 \ldots n} \frac{\left|x_{k}^{(0)}-\widetilde{x}_{k^{\mathrm{com}}}^{(0)}\right|}{x_{k}^{(0)}} \times 100 \%
$$

Undoubtedly, the MAPE has been an ideal indicator that can be used to estimate the forecasting accuracy of prediction models [32,33].

\section{Experimental Results}

Along with the constant energy consumption worldwide, energy consumption will rise massively by more than $50 \%$ before 2030 [34]. The development of effective prediction models for energy demand forecasting becomes increasingly significant, since energy demand forecasting has played an important role in energy management.

In this section, we conducted experiments using real data sequences to evaluate the forecasting accuracy of the proposed combined model. According to the parameter specifications specified by Ishibuchi and Tanaka [13], each network was implemented by a multi-layer perceptron (MLP) with a single input, one hidden layer with five units and a single output. The learning rate and momentum were specified as 0.25 and 0.9 , respectively. Section 5.1 briefly introduces the interval 
grey prediction models considered, and Section 5.2 demonstrates the forecasting accuracy for different prediction models.

\subsection{Compared Interval Grey Prediction Models}

Several interval grey prediction models were considered. Their distinct features are briefly introduced as follows.

\subsubsection{The Interval Grey Number Prediction Model}

The IGNPM [19] determined the upper wrapping sequence $\left(\hat{x}_{u, 1}^{(0)}, \hat{x}_{u, 2}^{(0)}, \ldots, \hat{x}_{u, n}^{(0)}\right)$ and lower wrapping sequence $\left(\hat{x}_{l, 1}^{(0)}, \hat{x}_{l, 2}^{(0)}, \ldots, \hat{x}_{l, n}^{(0)}\right)$ basis on the grey number layers. The area of the $k$-th grey number layer $s_{k}^{(0)}$ is defined as:

$$
s_{k}^{(0)}=\frac{x_{u, k}^{(0)}-x_{l, k}^{(0)}+x_{u, k+1}^{(0)}-x_{l, k+1}^{(0)}}{2}
$$

We can set up a GM(1,1) using the sequence $\left(s_{1}^{(0)}, s_{2}^{(0)}, \ldots, s_{n-1}^{(0)}\right)$, where

$$
\hat{s}_{k}^{(0)}=\left(1-e^{a_{s}}\right)\left(s_{1}^{(0)}-\frac{b_{s}}{a_{s}}\right) e^{-a_{s}(k-1)}, k=2,3, \ldots, n-1
$$

and then, with respect to $\hat{x}_{u, k}^{(0)}-\hat{x}_{l, k}^{(0)}$ :

$$
\hat{x}_{u, k}^{(0)}-\hat{x}_{l, k}^{(0)}=\frac{2\left(1-e^{a_{s}}\right)\left(s_{1}^{(0)}-\frac{b_{s}}{a^{s}}\right) e^{-a_{s}(k-2)}\left(1-\left(-e^{a_{s}}\right)^{k-2}\right)}{1+e^{a_{s}}}+(-1)^{k}\left(x_{u, 2}^{(0)}-x_{l, 2}^{(0)}\right)
$$

For the $k$-th grey number layer, a middle point $w_{k}^{(0)}$ is formulated as:

$$
w_{k}^{(0)}=\frac{x_{u, k}^{(0)}+x_{l, k}^{(0)}+x_{u, k+1}^{(0)}+x_{l, k+1}^{(0)}}{4}
$$

The sequence $\left(w_{1}^{(0)}, w_{2}^{(0)}, \ldots, w_{n-1}^{(0)}\right)$ is used to construct a $\operatorname{GM}(1,1)$ such that

$$
\hat{w}_{k}^{(0)}=\left(1-e^{a_{w}}\right)\left(w_{1}^{(0)}-\frac{b_{w}}{a_{w}}\right) e^{-a_{w}(k-1)}, k=2,3, \ldots, n-1
$$

and then, with respect to $\hat{x}_{u, k}^{(0)}+\hat{x}_{l, k}^{(0)}$ :

$$
\hat{x}_{u, k}^{(0)}+\hat{x}_{l, k}^{(0)}=\frac{4\left(1-e^{a_{w}}\right)\left(w_{1}^{(0)}-\frac{b_{w w}}{a_{w}}\right) e^{-a_{w v}(k-2)}\left(1-\left(-e^{a_{w}}\right)^{k-2}\right)}{1+e^{a_{w}}}+(-1)^{k}\left(x_{u, 2}^{(0)}+x_{l, 2}^{(0)}\right)
$$

The IGNPM ended up with the determination of $\hat{x}_{u, k}^{(0)}$ and $\hat{x}_{l, k}^{(0)}$ in terms of $\hat{x}_{u, k}^{(0)}-\hat{x}_{l, k}^{(0)}$ and $\hat{x}_{u, k}^{(0)}+\hat{x}_{l, k}^{(0)}$.

\subsubsection{The Grey Number Grey Modification Model}

A sequence $\mathbf{x}_{m}^{(0)}=\left(x_{m}^{(0)}, x_{m+1}^{(0)}, \ldots, x_{n}^{(0)}\right)=\left(x_{m, 1}^{(0)}, x_{m, 2}^{(0)} \ldots, x_{m, n-m+1}^{(0)}\right)(1 \leq \mathrm{m} \leq \mathrm{n}-3)$ was considered in the GGMM(1,1) [1], where $x_{m, 1}^{(0)}$ is replaced with $x_{n}^{(1)}$ to obtain $\hat{x}_{m, k}^{(0)}$ to derive the latest tendency [35]:

$$
\hat{x}_{m, k}^{(0)}=\left(1-e^{a_{m}}\right)\left(x_{n}^{(1)}-\frac{b_{m}}{a_{m}}\right) e^{-a_{m}(k-n)}, k=2,3, \ldots, n-m+1
$$


Since there are $m$ sequences, $\hat{x}_{u, k}^{(0)}$ and $\hat{x}_{l, k}^{(0)}$ can be formulated as

$$
\begin{aligned}
& \hat{x}_{u, k}^{(0)}=\max _{1 . . r}\left\{\hat{x}_{m, k}^{(0)}\right\} \\
& \hat{x}_{l, k}^{(0)}=\min _{1 . . r}\left\{\hat{x}_{m, k}^{(0)}\right\}
\end{aligned}
$$

where $r=\min \{k, m\}$. In particular, $a_{m}$ and $b_{m}$ are estimated by grey difference equations as

$$
x_{m, k}^{(0)}+a_{m} z_{k}^{(1)}=b_{m}
$$

where $z_{k}^{(1)}$ is:

$$
z_{k}^{(1)}=\frac{x_{k}^{(0)}}{\ln \frac{x_{k}^{(0)}}{x_{k-1}^{(0)}}}+x_{k}^{(1)}-\frac{\left(x_{k}^{(0)}\right)^{2}}{x_{k}^{(0)}-x_{k-1}^{(0)}}
$$

To sum up, the BNP values for IGNPM and GGMM(1,1) are the same as those for the IGF-NNs.

\subsection{Applications to Energy Demand Forecasting}

\subsubsection{Case I: Annual Electricity Demand of China}

China is the highest energy-using country in Asia, and the second largest economy in the world. Facing global warming, China's energy policy not only impacts its own sustainable development, but can also hugely influence global energy distribution. The first experiment considered the annual electricity demand, using available data from the China Statistical Yearbook 2016. Data from 2001-2012 were used for model fitting, and from 2013-2016 for ex post testing. Let $\alpha$ and $\beta$ range from zero to an arbitrarily large number of five, then the optimal combination of $\alpha$ and $\beta$ can make MAPE $\mathrm{C}_{\mathrm{com}}$ minimal. Such a combination can be determined by using the toolbox in MATLAB to implement a real-valued GA.

The forecasting accuracy of different prediction models is summarized in Tables 1 and 2. Results show that the proposed NN-FLNGM $(1,1)$ is promising as it was superior to the other forecasting models for ex post testing. Although the NN-FLNGM $(1,1)$ was slightly inferior to the $\operatorname{FLNGM}(1,1)$ for model fitting, the forecasting accuracy for ex post testing is the primary norm to examine the prediction

\begin{tabular}{|c|c|c|c|c|c|c|c|c|c|c|c|}
\hline \multirow{2}{*}{ Year } & \multirow{2}{*}{ Actual } & \multicolumn{2}{|c|}{ IGF-NN } & \multicolumn{2}{|c|}{ IGF-FLNGM(1,1) } & \multicolumn{2}{|c|}{ IGNPM } & \multicolumn{2}{|c|}{ GGMM(1,1) } & \multicolumn{2}{|c|}{ NN-FLNGM(1,1) } \\
\hline & & Predicted & APE & Predicted & APE & Predicted & APE & Predicted & APE & Predicted & APE \\
\hline 2001 & $14,633.46$ & $14,300.67$ & 2.27 & $14,300.67$ & 2.27 & $16,390.77$ & 12.01 & $14,633.46$ & 0.00 & $13,984.3$ & 4.44 \\
\hline 2002 & $16,331.45$ & $16,896.3$ & 3.46 & $16,878.96$ & 3.35 & $16,896.3$ & 3.46 & $17,100.29$ & 4.71 & $15,972.62$ & 2.20 \\
\hline 2003 & $19,031.6$ & $19,869.09$ & 4.40 & $21,423.59$ & 12.57 & $24,537.58$ & 28.93 & $20,285.82$ & 6.59 & $20,166.94$ & 5.97 \\
\hline 2004 & $21,971.38$ & $23,127.66$ & 5.26 & $23,944.65$ & 8.98 & $21,084.28$ & 4.04 & $22,267.95$ & 1.35 & $22,425.95$ & 2.07 \\
\hline 2005 & $24,940.32$ & $26,568.33$ & 6.53 & $26,895.6$ & 7.84 & $29,148.86$ & 16.87 & $23,979.10$ & 3.85 & $26,569.76$ & 6.53 \\
\hline 2006 & $28,587.97$ & $30,087.24$ & 5.24 & $30,283.08$ & 5.93 & $26,161.65$ & 8.49 & $26,167.56$ & 8.47 & $29,185.28$ & 2.09 \\
\hline 2007 & $37,211.8$ & $33,590.06$ & 9.73 & $33,799.86$ & 9.17 & $34,739.44$ & 6.64 & $31,033.86$ & 16.60 & $33,793.02$ & 9.19 \\
\hline 2008 & $34,541.35$ & $36,997.67$ & 7.11 & $37,230.13$ & 7.78 & $32,317.31$ & 6.44 & $35,095.77$ & 1.61 & $36,253.6$ & 4.96 \\
\hline 2009 & $37,032.14$ & $40,248.17$ & 8.68 & $40,492.88$ & 9.35 & $41,517.28$ & 12.11 & $36,746.24$ & 0.77 & $40,791.1$ & 10.15 \\
\hline 2010 & $41,934.49$ & $43,296.46$ & 3.25 & $43,162.98$ & 2.93 & $39,780.23$ & 5.14 & $39,545.96$ & 5.70 & $42,698.22$ & 1.82 \\
\hline 2011 & $47,000.88$ & $46,112.69$ & 1.89 & $45,088.18$ & 4.07 & $49,734.53$ & 5.82 & $43,678.58$ & 7.07 & $46,625.69$ & 0.80 \\
\hline 2012 & $49,762.64$ & $48,680.08$ & 2.18 & $48,321.47$ & 2.90 & $48,828.04$ & 1.88 & $48,243.27$ & 3.05 & $48,363.14$ & 2.81 \\
\hline
\end{tabular}
capability of a forecasting model.

Table 1. Forecasting accuracy of different interval grey number forecasting models for Case I (unit: 100 million kWh). 
Table 1. Cont.

\begin{tabular}{|c|c|c|c|c|c|c|c|c|c|c|c|}
\hline \multirow{2}{*}{ Year } & \multirow{2}{*}{ Actual } & \multicolumn{2}{|c|}{ IGF-NN } & \multicolumn{2}{|c|}{ IGF-FLNGM(1,1) } & \multicolumn{2}{|c|}{ IGNPM } & \multicolumn{2}{|c|}{ GGMM(1,1) } & \multicolumn{2}{|c|}{ NN-FLNGM(1,1) } \\
\hline & & Predicted & APE & Predicted & APE & Predicted & APE & Predicted & APE & Predicted & APE \\
\hline MAPE & & & 5.00 & & 6.43 & & 9.32 & & 4.98 & & 4.42 \\
\hline 2013 & $54,203.41$ & $50,992.52$ & 5.92 & $52,557.06$ & 3.04 & $59,696.86$ & 10.13 & $53,064.90$ & 2.10 & $52,782.04$ & 2.62 \\
\hline 2014 & $56,383.69$ & $53,052.18$ & 5.91 & $57,474.69$ & 1.93 & $59,797.33$ & 6.05 & $58,246.18$ & 3.30 & $54,718.01$ & 2.95 \\
\hline 2015 & $58,019.97$ & $54,867.18$ & 5.43 & $63,048.75$ & 8.67 & $71,774.88$ & 23.71 & $63,938.25$ & 10.20 & $59,289.04$ & 2.19 \\
\hline 2016 & $61,297.09$ & $56,449.38$ & 7.91 & $69,324.71$ & 13.10 & $73,096.15$ & 19.25 & $70,191.92$ & 14.51 & $61,306.91$ & 0.02 \\
\hline MAPE & & & 6.29 & & 6.68 & & 14.79 & & 7.53 & & 1.94 \\
\hline
\end{tabular}

Table 2. Forecasting accuracy of different prediction models for Case I (unit: 100 million kWh).

\begin{tabular}{cccccccccc}
\hline \multirow{2}{*}{ Year } & \multirow{2}{*}{ Actual } & \multicolumn{2}{c}{ NN } & & ARIMA & & \multicolumn{2}{c}{ GM(1,1) } & \multicolumn{2}{c}{ FLNGM(1,1) } \\
\cline { 3 - 9 } & & Predicted & APE & Predicted & APE & Predicted & APE & Predicted & APE \\
\hline 2001 & $14,633.46$ & $16,398.85$ & 12.06 & $14,633.46$ & 0.00 & $14,633.46$ & 0.00 & $14,633.46$ & 0.00 \\
2002 & $16,331.45$ & $17,533.93$ & 7.36 & $14,937.32$ & 8.54 & $18,481.63$ & 13.17 & $16,146.45$ & 1.13 \\
2003 & $19,031.6$ & $19,207.48$ & 0.92 & $18,984.30$ & 0.25 & $20,423.29$ & 7.31 & $18,932.71$ & 0.52 \\
2004 & $21,971.38$ & $21,518.08$ & 2.06 & $22,888.61$ & 4.17 & $22,568.94$ & 2.72 & $21,966.45$ & 0.02 \\
2005 & $24,940.32$ & $24,483.08$ & 1.83 & $26,655.28$ & 6.88 & $24,940.00$ & 0.00 & $25,281.11$ & 1.37 \\
2006 & $28,587.97$ & $28,004.94$ & 2.04 & $30,289.16$ & 5.95 & $27,560.17$ & 3.60 & $28,678.21$ & 0.32 \\
2007 & $37,211.8$ & $31,880.39$ & 14.33 & $33,794.93$ & 9.18 & $30,455.61$ & 18.16 & $32,017.3$ & 13.96 \\
2008 & $34,541.35$ & $35,855.23$ & 3.80 & $37,177.11$ & 7.63 & $33,655.24$ & 2.57 & $35,277.03$ & 2.13 \\
2009 & $37,032.14$ & $39,694.69$ & 7.19 & $40,440.06$ & 6.61 & $37,191.02$ & 0.43 & $38,487.54$ & 3.93 \\
2010 & $41,934.49$ & $43,231.85$ & 3.09 & $43,587.98$ & 3.94 & $41,098.26$ & 1.99 & $41,705.57$ & 0.55 \\
2011 & $47,000.88$ & $46,379.42$ & 1.32 & $46,624.93$ & 0.80 & $45,415.99$ & 3.37 & $45,239.89$ & 3.75 \\
2012 & $49,762.64$ & $49,114.49$ & 1.30 & $49,554.81$ & 0.42 & $50,187.34$ & 0.85 & $49,670.02$ & 0.19 \\
\hline MAPE & & & 4.78 & & 7.16 & & 4.51 & 2.32 \\
\hline 2013 & $54,203.41$ & $51,454.11$ & 5.07 & $52,381.41$ & 3.36 & $55,459.96$ & 2.32 & $55,111.25$ & 1.67 \\
2014 & $56,383.69$ & $53,434.01$ & 5.23 & $55,108.35$ & 2.26 & $61,286.52$ & 8.70 & $61,322.78$ & 8.76 \\
2015 & $58,019.97$ & $55,094.69$ & 5.04 & $57,739.17$ & 0.48 & $67,725.21$ & 16.73 & $68,209.12$ & 17.56 \\
2016 & $61,297.09$ & $56,474.52$ & 7.87 & $60,277.23$ & 1.66 & $74,840.33$ & 22.09 & $75,801.45$ & 23.66 \\
\hline MAPE & & & 5.80 & & 1.94 & & 12.46 & 12.91 \\
\hline
\end{tabular}

\subsubsection{Case II: Annual Energy Demand of Taiwan}

The second experiment considered annual energy demand using data from the Taiwan Energy Bureau. Data from 2001-2012 were selected for model fitting, and from 2013 and 2016 for ex post testing. The best combination of $\alpha$ and $\beta$ that make MAPE $\mathrm{com}_{\mathrm{cm}}$ minimal can be determined by a GA.

Tables 3 and 4 summarize model fitting and ex post testing results, respectively, for the different prediction models. Like Case 1, the proposed NN-FLNGM $(1,1)$ outperformed the other prediction models considered for ex post testing. Furthermore, experimental results show that the setting of ranges for $\alpha$ and $\beta$ are acceptable. The ranges of $\alpha$ and $\beta$ seem not be a serious problem to set up the proposed prediction model. It is noted that the Autoregressive Integrated Moving Average model (ARIMA) ARIMA $(1,0,1)$ fits the statistical properties of available data well for both Cases I and II, where $\operatorname{ARIMA}(p, d, q)$ denotes that the autoregressive model of order $p$, the moving average model of order $q$ and the number of differences $d$ are taken into account. 
Table 3. Forecasting accuracy of different interval grey number forecasting models for Case II (unit: $104 \mathrm{kLOE})$.

\begin{tabular}{|c|c|c|c|c|c|c|c|c|c|c|c|}
\hline \multirow{2}{*}{ Year } & \multirow{2}{*}{ Actual } & \multicolumn{2}{|c|}{ IGF-NNs } & \multicolumn{2}{|c|}{ IGF-FLNGM(1,1) } & \multicolumn{2}{|c|}{ IGNPM } & \multicolumn{2}{|c|}{ GGMM(1,1) } & \multicolumn{2}{|c|}{ NN-FLNGM(1,1) } \\
\hline & & Predicted & APE & Predicted & APE & Predicted & APE & Predicted & APE & Predicted & APE \\
\hline 2001 & $91,333.4$ & $92,222.6$ & 0.97 & $92,222.6$ & 0.97 & $92,222.6$ & 0.97 & $91,333.4$ & 0.00 & $90,748.7$ & 0.64 \\
\hline 2002 & $95,385.9$ & $96,977.9$ & 1.67 & $97,293.0$ & 2.00 & $96,977.9$ & 6.48 & $97,463.4$ & 2.18 & $95,632.9$ & 0.26 \\
\hline 2003 & $99,252.5$ & $100,972.1$ & 1.73 & $102,674.8$ & 3.45 & $107,376.6$ & 3.63 & $100,689.8$ & 1.45 & $100,655.9$ & 1.41 \\
\hline 2004 & $103,553.3$ & $104,180.7$ & 0.61 & $104,082.3$ & 0.51 & $99,487.7$ & 0.59 & $104,298.3$ & 0.72 & $103,020.0$ & 0.51 \\
\hline 2005 & $105,700.9$ & $106,626.1$ & 0.88 & $105,739.0$ & 0.04 & $109,917.2$ & 0.20 & $106,626.8$ & 0.88 & $105,504.0$ & 0.19 \\
\hline 2006 & $107,773.8$ & $108,398.4$ & 0.58 & $107,027.0$ & 0.69 & $102,059.5$ & 0.87 & $109,862.2$ & 1.94 & $107,094.1$ & 0.63 \\
\hline 2007 & $113,024.6$ & $109,638.7$ & 3.00 & $108,561.3$ & 3.95 & $112,520.6$ & 4.27 & $110,826.3$ & 1.95 & $108,923.2$ & 3.63 \\
\hline 2008 & $109,819.2$ & $110,493.4$ & 0.61 & $109,684.5$ & 0.12 & $104,694.8$ & 0.23 & $106,287.3$ & 3.22 & $109,969.0$ & 0.14 \\
\hline 2009 & 107,677 & $111,082.4$ & 3.16 & $110,919.9$ & 3.01 & $115,188.4$ & 3.05 & $100,195.6$ & 6.95 & $111,332.6$ & 3.40 \\
\hline 2010 & 114,368 & $111,492.4$ & 2.51 & $111,582.0$ & 2.44 & $107,395.3$ & 1.74 & $143,562.3$ & 25.53 & $111,927.7$ & 2.13 \\
\hline 2011 & $113,105.3$ & $111,781.6$ & 1.17 & $112,783.4$ & 0.28 & $117,922.0$ & 0.62 & $113,244.6$ & 0.12 & $113,129.0$ & 0.02 \\
\hline 2012 & $112,870.8$ & $111,988.6$ & 0.78 & $113,733.0$ & 0.76 & $110,162.6$ & 2.11 & $114,043.2$ & 1.04 & $113,758.1$ & 0.79 \\
\hline MAPE & & & 1.47 & & 1.52 & & 4.07 & & 3.83 & & 1.15 \\
\hline 2013 & $115,893.7$ & $112,138.8$ & 3.24 & $115,153.0$ & 0.64 & $120,723.2$ & 4.17 & $114,553.4$ & 1.16 & $115,002.4$ & 0.77 \\
\hline 2014 & $116,826.5$ & $112,249.1$ & 3.92 & $116,136.7$ & 0.59 & $112,998.2$ & 3.28 & $115,079.3$ & 1.50 & $115,597.1$ & 1.05 \\
\hline 2015 & $116,509.1$ & $112,330.8$ & 3.59 & $117,615.5$ & 0.95 & $123,593.7$ & 6.08 & $115,621.2$ & 0.76 & $116,843.3$ & 0.29 \\
\hline 2016 & $116,808.9$ & $112,391.9$ & 3.78 & $118,940.7$ & 1.82 & $115,903.9$ & 0.77 & $116,179.3$ & 0.54 & $117,578.7$ & 0.66 \\
\hline MAPE & & & 3.63 & & 1.00 & & 3.57 & & 0.99 & & 0.69 \\
\hline
\end{tabular}

Table 4. Forecasting accuracy of different prediction models for Case II (unit: $104 \mathrm{kLOE})$.

\begin{tabular}{|c|c|c|c|c|c|c|c|c|c|}
\hline \multirow{2}{*}{ Year } & \multirow{2}{*}{ Actual } & \multicolumn{2}{|c|}{$\mathbf{N N}$} & \multicolumn{2}{|l|}{ ARIMA } & \multicolumn{2}{|c|}{ GM(1,1) } & \multicolumn{2}{|c|}{ FLNGM(1,1) } \\
\hline & & Predicted & APE & Predicted & APE & Predicted & APE & Predicted & APE \\
\hline 2001 & $91,333.4$ & $90,894.3$ & 0.48 & $91,333.4$ & 4.24 & $91,333.4$ & 0.00 & $91,333.4$ & 0.00 \\
\hline 2002 & $95,385.9$ & $95,719.4$ & 0.35 & $95,203.1$ & 4.60 & $100,267.5$ & 5.12 & $95,315.22$ & 0.07 \\
\hline 2003 & $99,252.5$ & $99,957.2$ & 0.71 & $99,774.9$ & 4.03 & $101,798.4$ & 2.57 & $100,601.9$ & 1.36 \\
\hline 2004 & $103,553.3$ & $103,408.2$ & 0.14 & $103,256.6$ & 2.27 & $103,352.8$ & 0.19 & $103,278.7$ & 0.27 \\
\hline 2005 & $105,700.9$ & $106,103.0$ & 0.38 & $105,907.9$ & 2.11 & $104,930.8$ & 0.73 & $105,702.5$ & 0.00 \\
\hline 2006 & $107,773.8$ & $108,158.0$ & 0.36 & $107,927.1$ & 1.57 & $106,533.0$ & 1.15 & $107,747.7$ & 0.02 \\
\hline 2007 & $113,024.6$ & $109,704.4$ & 2.94 & $109,464.7$ & 2.11 & $108,159.6$ & 4.30 & $109,379.3$ & 3.23 \\
\hline 2008 & $109,819.2$ & $110,859.7$ & 0.95 & $110,635.7$ & 1.56 & $109,811.1$ & 0.01 & $110,579.4$ & 0.69 \\
\hline 2009 & 107,677 & $111,719.6$ & 3.75 & $111,527.4$ & 4.21 & $111,487.7$ & 3.54 & $111,368.7$ & 3.43 \\
\hline 2010 & 114,368 & $112,358.6$ & 1.76 & $112,206.5$ & 1.44 & $113,190.0$ & 1.03 & $111,885.9$ & 2.17 \\
\hline 2011 & $113,105.3$ & $112,833.4$ & 0.24 & $112,723.6$ & 0.01 & $114,918.3$ & 1.60 & $112,392.1$ & 0.63 \\
\hline 2012 & $112,870.8$ & $113,186.5$ & 0.28 & $113,117.5$ & 0.48 & $116,672.9$ & 3.37 & $113,147.5$ & 0.25 \\
\hline MAPE & & & 1.03 & & 7.16 & & 1.97 & & 1.01 \\
\hline 2013 & $115,893.7$ & $113,449.6$ & 2.11 & $113,417.4$ & 1.79 & $118,454.36$ & 2.21 & $114,310.1$ & 1.37 \\
\hline 2014 & $116,826.5$ & $113,645.9$ & 2.72 & $113,645.8$ & 2.72 & $120,263.00$ & 2.94 & $115,957.9$ & 0.74 \\
\hline 2015 & $116,509.1$ & $113,792.8$ & 2.33 & $113,819.7$ & 2.31 & $122,099.26$ & 4.80 & $118,145.1$ & 1.40 \\
\hline 2016 & $116,808.9$ & $113,903.0$ & 2.49 & $113,952.2$ & 2.45 & $123,963.56$ & 6.13 & $120,887.8$ & 3.49 \\
\hline MAPE & & & 2.41 & & 2.40 & & 4.02 & & 1.75 \\
\hline
\end{tabular}

\section{Discussion and Conclusions}

As for China, it is mainly satisfied by fossil fuels [36], while a massive need for oil, coal and gas can be expected, along with rapid economic growth [4,37]. Undoubtedly, the development of prediction models for energy demand forecasting is very crucial for future economic prosperity and environmental security. Due to the uncertain and imprecise nature of the available energy demand data, it is reasonable to develop the interval grey prediction models for energy demand forecasting. As a matter of fact, energy demand forecasting can be treated as a kind of grey system problems $[4,38]$, since factors such as gross domestic product, population, urbanization rate and the share of coal energy could affect energy demand [39]. Nevertheless, it is not clear what the precise manner of the impact is.

This study used a simplified version of fuzzy regression analysis [40,41], interval regression analysis, to estimate interval grey numbers. Because available data usually exhibit nonlinear tendencies, 
IGF-NNs created by two NNs were considered. Although data uncertainty can be dealt with by interval regression analysis, it is interesting to further examine the prediction capability of the IGF-NNs by defuzzifying interval grey numbers derived by the IGF-NNs. It is also possible to improve IGF-NN prediction accuracy further by combining results obtained by the other interval grey number prediction model. Thus, this study applied interval grey numbers obtained by the IGF-NNs to construct the IGF-FLNGM $(1,1)$, and then a combined BNP for $x_{k}^{(0)}$ was calculated by a weighted average of BNP values from the IGF-NNs and IGF-FLNGM(1,1).

The forecasting accuracy of the proposed combined model was evaluated by real data collected from public sectors. The combined model exhibited superior prediction accuracy compared with the other prediction models considered for ex post testing. The proposed model also outperformed the other interval grey prediction models considered, including IGNPM, GGMM(1,1), IGF-NNs and IGF-FLNGM(1,1), for both model fitting and ex post testing. This further confirms that the forest combination improves point forecast performance. It is noted that the proposed combined model is quite different from the IGNPM and IN-DGM, as it applies nonlinear regression analysis to determine the upper and lower wrapping sequences automatically based on real-valued data sequences. In other words, the proposed combined model is driven by the real-valued sequences, while the IGNPM and the IN-DGM are mainly driven by interval-valued ones.

For Taiwan, almost $98 \%$ of energy was imported, reaching 13-15\% of gross domestic product, and energy supply is highly dependent on imported fossil fuel, which is the leading cause of high carbon dioxide emissions. The results obtained by the proposed combined model were very encouraging regarding predicting Taiwan energy demand, and the government could make use of the proposed combined model to set up energy plans to promote environmental protection and sustainable economic growth.

As for the future work, the activation function in the FLN computed the weighted sum of a weight vector with an input. However, the interaction among variables was not considered in the weighted sum. Since variables are likely to be dependent on each other [42-44], our future work will investigate how to incorporate the nonadditive $\operatorname{FLNGM}(1,1)[45]$ into the proposed combined model.

Author Contributions: Conceptualization, Y.-C.H. and P.J.; methodology, Y.-C.H. and W.W.; formal analysis, H.J.; data curation, G.W.; writing-original draft preparation, Y.-C.H. and P.J.; writing-review \& editing, Y.-C.H. and P.J.; funding acquisition, Y.-C.H. and P.J. All authors have read and agreed to the published version of the manuscript.

Funding: This research is supported by the Ministry of Science and Technology, Taiwan, under Grant MOST 108-2410-H-033-038-MY2; the Youth Team Grant Fund of Shandong University and Business School; the Culture Image and Competitiveness of Tourism in China under Grant 2018WQTDXM003 provided additional funding; and the research project of Humanities and Social Sciences of Shandong University (No. IFWF2029).

Acknowledgments: The authors would like to thank the anonymous referees for their valuable comments.

Conflicts of Interest: The authors declare no conflict of interest.

\section{References}

1. Shih, C.-S.; Hsu, Y.-T.; Yeh, J.; Lee, P.-C. Grey number prediction using the grey modification model with progression technique. Appl. Math. Model. 2011, 35, 1314-1321. [CrossRef]

2. $\mathrm{Hu}, \mathrm{Y} . \mathrm{C}$. Electricity consumption forecasting using a neural-network-based grey prediction approach. J. Oper. Res. Soc. 2017, 68, 1259-1264. [CrossRef]

3. Lee, Y.-S.; Tong, L.-I. Forecasting energy consumption using a grey model improved by incorporating genetic programming. Energy Convers. Manag. 2011, 52, 147-152. [CrossRef]

4. Suganthi, L.; Samuel, A.A. Energy models for demand forecasting-A review. Renew. Sustain. Energy Rev. 2012, 16, 1223-1240. [CrossRef]

5. Hwang, C.; Hong, D.H.; Seok, K.H. Support vector interval regression machine for crisp input and output data. Fuzzy Sets Syst. 2006, 157, 1114-1125. [CrossRef]

6. Xie, N.; Liu, S.; Yuan, C.; Yang, Y. Grey number sequence forecasting approach for interval analysis: A case of China's gross domestic product prediction. J. Grey Syst. 2014, 26, 45-58. 
7. Zeng, B.; Li, C.; Zhou, X.-Y.; Long, X.-J. Prediction Model of Interval Grey Numbers with a Real Parameter and Its Application. Abstr. Appl. Anal. 2014, 2014,1-12. [CrossRef]

8. Huang, L.; Zhang, B.-L.; Huang, Q. Robust interval regression analysis using neural networks. Fuzzy Sets Syst. 1998, 97, 337-347. [CrossRef]

9. Jeng, J.-T.; Chuang, C.-C.; Su, S.-F. Support vector interval regression networks for interval regression analysis. Fuzzy Sets Syst. 2003, 138, 283-300. [CrossRef]

10. Cheng, C.-B.; Lee, E.S. Fuzzy regression with radial basis function network. Fuzzy Sets Syst. 2001, 119, 291-301. [CrossRef]

11. Hu, Y.-C. Functional-link nets with genetic-algorithm-based learning for robust nonlinear interval regression analysis. Neurocomputing 2009, 72, 1808-1816. [CrossRef]

12. Ishibuchi, H.; Nii, M. Fuzzy regression using asymmetric fuzzy coefficients and fuzzified neural networks. Fuzzy Sets Syst. 2001, 119, 273-290. [CrossRef]

13. Ishibuchi, H.; Tanaka, H. Fuzzy regression analysis using neural networks. Fuzzy Sets Syst. 1992, 50, $257-265$. [CrossRef]

14. Sun, X.; Sun, W.; Wang, J.; Zhang, Y.; Gao, Y. Using a Grey-Markov model optimized by Cuckoo search algorithm to forecast the annual foreign tourist arrivals to China. Tour. Manag. 2016, 52, 369-379. [CrossRef]

15. Deng, J.L. Control problems of grey systems. Syst. Control Lett. 1982, 1, 288-294.

16. Liu, S.; Lin, Y. Grey Information: Theory and Practical Applications; Springer: London, UK, 2010; pp. $93-101$.

17. Liu, S.; Yang, Y.; Forrest, J. Grey Data Analysis: Methods, Models and Applications; Springer: Berlin, Germany, 2017.

18. Xu, N.; Dang, Y.; Gong, Y. Novel grey prediction model with nonlinear optimized time response method for forecasting of electricity consumption in China. Energy 2017, 118, 473-480. [CrossRef]

19. Zeng, B.; Liu, S.; Xie, N.; Cui, J. Prediction model for interval grey number based on grey band and grey layer. Control Decis. 2010, 25, 1585-1592.

20. Zeng, B.; Liu, S.; Xie, N. Prediction model of interval grey number based on DGM $(1,1)$. J. Syst. Eng. Electron. 2010, 21, 598-603. [CrossRef]

21. Chen, Y.; He, K.; Zhang, C. A novel grey wave forecasting method for predicting metal prices. Resour. Policy 2016, 49, 323-331. [CrossRef]

22. Li, K.; Liu, L.; Zhai, J.; Khoshgoftaar, T.M.; Li, T. The improved grey model based on particle swarm optimization algorithm for time series prediction. Eng. Appl. Artif. Intell. 2016, 55, 285-291. [CrossRef]

23. Zhao, H.; Guo, S. An optimized grey model for annual power load forecasting. Energy 2016, 107, $272-286$. [CrossRef]

24. Hsu, L.-C. Applying the Grey prediction model to the global integrated circuit industry. Technol. Forecast. Soc. Chang. 2003, 70, 563-574. [CrossRef]

25. Hsu, C.-C.; Chen, C.-Y. Applications of improved grey prediction model for power demand forecasting. Energy Convers. Manag. 2003, 44, 2241-2249. [CrossRef]

26. Hsu, C.-I.; Wen, Y. Improved grey prediction models for the trans?pacific air passenger market. Transp. Plan. Technol. 1998, 22, 87-107. [CrossRef]

27. $\mathrm{Hu}, \mathrm{Y} .-\mathrm{C}$. Grey prediction with residual modification using functional-link net and its application to energy demand forecasting. Kybernetes 2017, 46, 349-363. [CrossRef]

28. Cang, S. A Comparative Analysis of Three Types of Tourism Demand Forecasting Models: Individual, Linear Combination and Non-linear Combination. Int. J. Tour. Res. 2013, 16, 596-607. [CrossRef]

29. Clemen, R.T. Combining forecasts: A review and annotated bibliography. Int. J. Forecast. 1989, 5, 559-583. [CrossRef]

30. Wu, D.C.; Song, H.; Shen, S. New developments in tourism and hotel demand modeling and forecasting. Int. J. Contemp. Hosp. Manag. 2017, 29, 507-529. [CrossRef]

31. Tzeng, G.-H.; Huang, J.-J. Fuzzy Multiple Objective Decision Making; Chapman and Hall/CRC Press: London, UK, 2016.

32. Lee, S.-C.; Shih, L.-H. Forecasting of electricity costs based on an enhanced gray-based learning model: A case study of renewable energy in Taiwan. Technol. Forecast. Soc. Chang. 2011, 78, 1242-1253. [CrossRef]

33. Makridakis, S. Accuracy measures: Theoretical and practical concerns. Int. J. Forecast. 1993, 9, $527-529$. [CrossRef] 
34. Smith, M.; Hargroves, K.; Stasinopoulos, P.; Stephens, R.; Desha, C.; Hargroves, S. Energy Transformed: Sustainable Energy Solutions for Climate Change Mitigation; Natural Edge Project; CSIRO and Griffith University: Queensland, Australia, 2007.

35. Dang, Y.; Liu, S.; Chen, K. The GM models that x(n) be taken as initial value. Kybernetes 2004, 5, 247-254.

36. National Bureau of Statistics of China. China Statistical Yearbook 2016; China Statistics Press: Beijing, China, 2016.

37. Adams, F.G.; Shachmurove, Y. Modeling and forecasting energy consumption in China: Implications for Chinese energy demand and imports in 2020. Energy Econ. 2008, 30, 1263-1278. [CrossRef]

38. Pi, D.; Liu, J.; Qin, X. A Grey Prediction Approach to Forecasting Energy Demand in China. Energy Sources Part A Recover. Util. Environ. Eff. 2010, 32, 1517-1528. [CrossRef]

39. Yu, S.; Wei, Y.-M.; Wang, K. China's primary energy demands in 2020: Predictions from an MPSO-RBF estimation model. Energy Convers. Manag. 2012, 61, 59-66. [CrossRef]

40. Tanaka, H. Fuzzy data analysis by possibilistic linear models. Fuzzy Sets Syst. 1987, 24, 363-375. [CrossRef]

41. Tanaka, H.; Uejima, S.; Asai, K. Linear regression analysis with fuzzy model. IEEE Trans. Syst. Man Cybern. 1982, 12, 903-907.

42. Hu, Y.-C. Nonadditive similarity-based single-layer perceptron for multi-criteria collaborative filtering. Neurocomputing 2014, 129, 306-314. [CrossRef]

43. Onisawa, T.; Sugeno, M.; Nishiwaki, Y.; Kawai, H.; Harima, Y. Fuzzy measure analysis of public attitude towards the use of nuclear energy. Fuzzy Sets Syst. 1986, 20, 259-289. [CrossRef]

44. Wang, W.; Wang, Z.; lir, K.G.J. Genetic algorithms for determining fuzzy measures from data. J. Intell. Fuzzy Syst. 1998, 6, 171-183.

45. Hu, Y.-C. Nonadditive Grey Prediction Using Functional-Link Net for Energy Demand Forecasting. Sustainability 2017, 9, 1166. [CrossRef]

(C) 2020 by the authors. Licensee MDPI, Basel, Switzerland. This article is an open access article distributed under the terms and conditions of the Creative Commons Attribution (CC BY) license (http://creativecommons.org/licenses/by/4.0/). 